\title{
A ROADMAP TO THE STANDARDIZATION OF IN VIVO MAGNETIC HYPERTHERMIA
}

\author{
L. Beola ${ }^{a}$, L. Gutiérrez ${ }^{b .}{ }^{*}$, V. Grazúa ${ }^{a}$, L. Asín ${ }^{a,{ }^{*}}$
}

\begin{abstract}
a Institute of Materials Science of Aragón, Universidad de Zaragoza, CSIC and CIBER-BBN, Campus Río Ebro, Edificio I+D, Mariano Esquillor Gómez, 50018 Zaragoza, Spain

${ }^{b}$ Department of Analytical Chemistry, Universidad de Zaragoza and CIBER$B B N$, Instituto Universitario de Nanociencia de Aragón (INA), Edificio I+D, Mariano Esquillor Gómez, 50018 Zaragoza, Spain
\end{abstract}

*Corresponding authors: LG (lu@unizar.es), LA (lasin@unizar.es).

\section{ABSTRACT}

Despite the fact that magnetic hyperthermia seems to be a promising approach in cancer treatment, researchers working in this field have to face several drawbacks, as the lack of standardization in the treatment conditions and the difficulties measuring the biological effects. This chapter revises recent bibliography about in vivo pre-clinical studies and gives an overview of the current state of the art in this topic. Several aspects of the methodology are discussed, like the type of magnetic nanoparticles most commonly used, parameters of the application of the magnetic field, animal and tumor models, techniques used to follow the tumor growth and the evaluation of the treatment effectiveness. The comparison and critical argumentation of all these points are expected to shed some light on the field of magnetic hyperthermia and to show the current tendencies in this kind of experiments.

KEYWORDS: In vivo, Magnetic nanoparticles, Magnetic hyperthermia, Animal models, Tumor models.

\section{Introduction}

The heating of tumors by means of Magnetic Hyperthermia (MH) presents clear advantages over conventional hyperthermia techniques (e.g.: ultrasounds, microwaves and radiofrequency), as it is not limited by tissue depth, obstruction 
or reflection of the energy by bone structures and heat-sink effects near large blood vessels. In addition, other advantages include a more homogeneous temperature distribution in the tumor mass, a reduction of the treatment invasiveness while enhancing its specificity, and the fact that the treatment can be carried out at cellular level instead of at tissue or organ level $(1,2)$.

The first phase I trial showing the feasibility of $\mathrm{MH}$ in human patients was carried out at Berlin Charité Hospital (2003 to 2005). Since then, several clinical trials mostly on recurrent tumors (e.g. glioblastoma multiforme, prostate, esophagus, liver cancer) have shown promising results, not only in terms of systemic toxicity and morbidity but also in the improvement of the median overall survival of patients (1). Specifically, in the case of glioblastoma, results obtained when $\mathrm{MH}$ was applied in combination with radiotherapy in 59 patients showed a median overall survival 10.6-16.2 months longer than the typical 6month survival rate in these patients (3). It is worth noting that these clinical studies started only 10 years after the group headed by Andreas Jordan initiated this field of $\mathrm{MH}(4)$ and not many pre-clinical studies were performed before that.

Despite these promising results, there are still several challenges to overcome for the establishment of $\mathrm{MH}$ in the clinical routine. In fact, many actual preclinical trials on $\mathrm{MH}$ are still trying to understand the importance of all the relevant parameters including physical, chemical, engineering, technological and biological aspects of this complex therapy strategy. Still, many knowledge gaps exist in the frame of in vivo $\mathrm{MH}$ applications. Of special relevance are the underlying physical mechanisms for heat generation, the cytotoxicity mechanisms that are triggered, the effect that the interaction of the magnetic nanoparticles (MNP) with biological media/tissues and cells have on the MNP heat efficiency, etc. In addition, there are several difficulties to reach the effective concentration in tumors when MNP are administered through intravenous routes (5). In this sense, it is important to highlight that in all the human clinical trials MNP were injected intratumorally.

Another big obstacle that the research community involved in $\mathrm{MH}$ studies faces in the development of this technique is the general lack of standardization of the experimental procedures, which makes very difficult to compare results among different laboratories. In this context, first of all, standardization is required for the scaled-up production of MNP with optimal magnetic characteristics for $\mathrm{MH}$. This will imply to overcome the long scientific and technological challenge of developing synthetic methodologies with a good control of the particle size, size distribution, shape and crystal defects (6). Another aspect that needs standardization is the fabrication and use of adequate alternating magnetic field (AMF) applicators. Hereof, there is still discussion about which is the best measurement methodology (calorimetric or magnetic measurements) and the 
best experimental conditions (adiabatic or non-adiabatic) to determine the heating efficiency in terms of the specific absorption rate (SAR) of the MNP. This is also a crucial aspect as the higher the SAR, the lower the injected dose of MNP needed for the patient treatment (7). Besides, as SAR values strongly depend on the field amplitude generated by AMF generators, the calibration of $\mathrm{MH}$ setups in order to obtain results that could be compared among different models and different laboratories is fundamental (8). Other aspects to be taken into account, besides the several well-known physical factors that influence the heating efficiency (e.g. amplitude $(\mathrm{H})$ and frequency (f) of the AMF, magnetic anisotropy, magnetization, particle-particle interactions, size and size distribution of the MNP, etc.), are biological issues. It has been reported that SAR values could shrink between $50 \%$ and $90 \%$ when either biomolecules are adsorbed onto the surface of MNP or MNP are located inside cells or tissues (8). Therefore, it is recommended to check the actual magnetic response of MNP in true biological environments and physiological conditions in order to understand the underlying reasons of the reduction of their magnetic heating (e.g. viscosity increase, aggregation) and thus engineer MNP with robust heating efficiency regardless of the host medium. For this reasons, besides the need of more experimentation using in vitro models that mimic better the tumor and its microenvironment (3D cell cultures, tumor spheroids, etc.), the role of computational simulated models of in vivo heat dissipation will be also essential for the development of more reliable treatment planning.

Other important challenge of $\mathrm{MH}$ is to control heat generation in order to achieve a uniform thermal profile within the tumor. This strongly depends on the homogeneity of MNP distribution inside the tumor and the route of administration. Thus, the development of efficient methods to selectively and uniformly deliver nanoparticles to the tumor is also an important area of research. So far, the administration mode selected in most in vivo studies is the intratumoral injection. However, this approach is not suitable for deep-seated and metastatic tumors. Moreover, intratumoral injection may cause tumor incongruence, invasiveness, and recurrence, especially from under-treated tumor regions due to a non-homogeneous temperature distribution.

A number of review articles and book chapters have already dealt with the challenges mentioned above in order to transfer $\mathrm{MH}$ to the clinical practice. An analysis of $\mathrm{MH}$ literature illustrated in Fig. 12.1, shows that more than 3000 articles were published from 1997 to date, dealing not only with the synthesis of adequate MNP but also with the underlying physical factors that influence heating efficiency. Fewer articles (around 500) were published dealing with in vitro $\mathrm{MH}$ experimentation including the biological issues that affect SAR. Both aspects of $\mathrm{MH}$ have followed an exponential growth at the beginning of the $21^{\text {st }}$ century. 

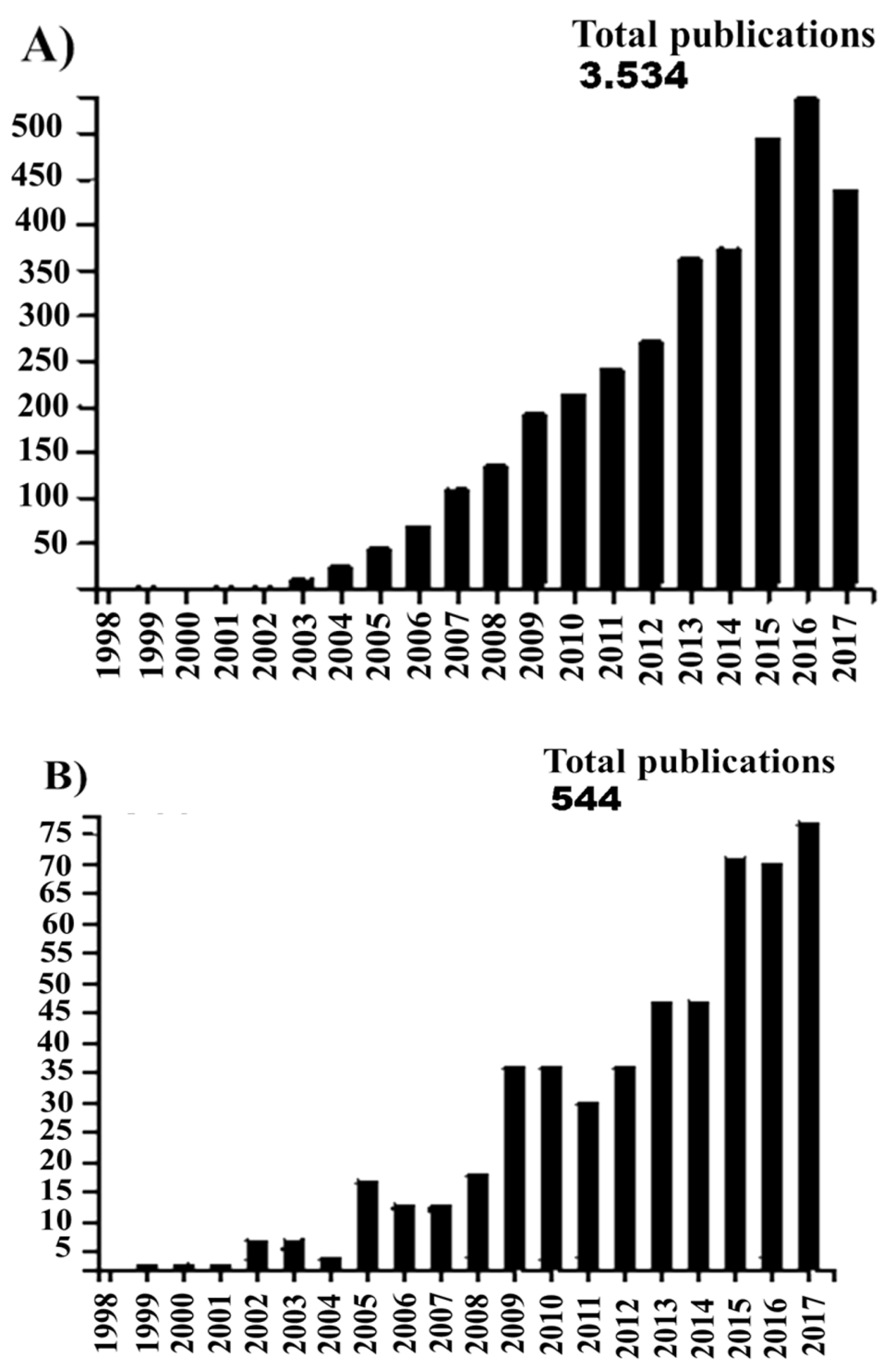

Fig. 12.1. Number of published articles during the period 1997-2017 using the following search terms: A) "magnetic nanoparticles and magnetic hyperthermia", and B) "in vitro studies and magnetic hyperthermia".

The number of articles reporting in vivo $\mathrm{MH}$ experimentation is however low, and it is difficult to find an overview that includes a comparison in terms of type and quantity of MNP, AMF applicator, AMF conditions, MNP administration mode, tumor, animal model, and methodologies used to follow the biological effect of $\mathrm{MH}$. This is the reason why this chapter focuses on the analysis of in vivo studies for pre-clinical applications of $\mathrm{MH}$ reported in the literature.

We have selected 33 studies published between 2001 and 2017, most of them are in vivo animal studies with only one research work on humans, and we compare the main relevant materials and methods used in all of them (Table 12.1). In addition, we discuss the advantages and disadvantages of the different 
animal models and the methodologies used to measure the in vivo biological effects of $\mathrm{MH}$. Lastly, as final remarks, we adress key issues where consensus must be reached by the $\mathrm{MH}$ community to make real progress towards clinical acceptance and implementation of $\mathrm{MH}$ in the clinical practice.

\begin{tabular}{|c|c|c|c|c|c|c|c|c|c|}
\hline & \multicolumn{4}{|c|}{ MNP } & \multicolumn{5}{|c|}{ Animal model } \\
\hline Ref. & Core & $\begin{array}{l}\text { Size } \\
(\mathrm{nm})\end{array}$ & $\begin{array}{c}\text { SAR } \\
\left(W^{-1}\right)\end{array}$ & $\begin{array}{c}H^{*} \mathbf{f} \\
\left(A m^{-1} s^{-1}\right)\end{array}$ & Animal & Tumor & $\begin{array}{l}\text { MNP } \\
\text { adm. }\end{array}$ & $\begin{array}{l}\text { Technique } \\
\text { used to } \\
\text { follow } \\
\text { tumor } \\
\text { growth }\end{array}$ & Effect \\
\hline (9) & 10 & 15 & ND & $1.810^{9}$ & $\mathrm{HCT}$ & $A, O$ & IT & $\mathrm{I}(\mathrm{CT})$ & $S R$ \\
\hline (10) & IC & 12 & 64 & $1.8310^{9}$ & M IC & $A, H$ & $\begin{array}{l}\text { IT and } \\
\text { IV }\end{array}$ & C & GI \\
\hline (11) & 10 & $<20$ & ND & $1.0810^{9}$ & Rab IC & $A, O$ & SM & Other & GI \\
\hline (12) & IC & $\begin{array}{c}5-10 x \\
1-4\end{array}$ & 620 & $1.8310^{9}$ & M IC & $A, H$ & IV & C & GI \\
\hline (13) & 10 & 15,12 & 658,900 & $6.710^{9}$ & M ID & $\mathrm{X}, \mathrm{H}$ & IT & $C$ & $\mathrm{Gl}$ \\
\hline (14) & 10 & 12 & 500 & $6.710^{9}$ & M ID & $\mathrm{X}, \mathrm{H}$ & IT & $C$ & GI or $\mathrm{CR}$ \\
\hline (15) & 10 & $20-50$ & ND & $5.910^{9}$ & M ID & $\mathrm{X}, \mathrm{H}$ & IT & C & $\mathrm{Gl}$ \\
\hline (16) & 10 & 30 & 2614 & $6.610^{9}$ & M ID & $\mathrm{X}, \mathrm{H}$ & IT & C & $\mathrm{GI}$ \\
\hline (17) & 10 & 19 & 500 & $8.8410^{9}$ & Rat IC & $A, O$ & IA & $I(U)$ & $\mathrm{GI}$ \\
\hline (18) & IC & 15 & ND & $1.2610^{9}$ & Rat IC & $A, O$ & IT & $I(U)$ & $\mathrm{GI}$ \\
\hline (19) & 10 & 12 & ND & $9.8410^{9}$ & M ID & $\mathrm{X}, \mathrm{H}$ & IT & $C$ & GI \\
\hline (20) & 10 & 19 & 137 & $2.6410^{9}$ & M ID & $\mathrm{X}, \mathrm{H}$ & IT & $\mathrm{C}$ & $\mathrm{GI}$ \\
\hline (21) & IC & $N P$ & 2,7 & $1.8710^{9}$ & M ID & $x, 0$ & IT & $\mathrm{C}, \mathrm{I}$ & $\mathrm{GI}$ \\
\hline (22) & 10 & 12 & 94 & $7.7010^{9}$ & M IC & PT, $\mathrm{H}$ & IT & ND & HSP 70 \\
\hline (23) & 10 & ND & 29 & $4.2310^{9}$ & M ID & $\mathrm{X}, \mathrm{H}$ & IV & $\mathrm{C}, \mathrm{I}$ & $\mathrm{GI}$ \\
\hline (24) & 10 & ND & ND & ND & Rat IC & $A, O$ & IT & I (MRI) & $\mathrm{GI}$ \\
\hline (25) & Other & 19 & 564 & $1.1210^{9}$ & M ID & $x, 0$ & IT & I (Fluo) & $\mathrm{GI}$ \\
\hline (26) & Other & 9 & ND & $7.5810^{9}$ & M ID & $\mathrm{X}, \mathrm{H}$ & IT & $\mathrm{C}, \mathrm{I}(\mathrm{MRI})$ & $\mathrm{GI}$ \\
\hline (27) & Other & 9 & ND & $4.8710^{9}$ & M ID & $\mathrm{X}, \mathrm{H}$ & IT & $\mathrm{C}, \mathrm{I}(\mathrm{MRI})$ & $\mathrm{GI}$ \\
\hline (28) & 10 & ND & 210 & $8.1810^{9}$ & M IC & $\mathrm{A}, \mathrm{H}$ & IT & $\mathrm{C}$ & $C R$ \\
\hline (29) & 10 & $10 \circ 12$ & ND & ND & Rab IC & $A, O$ & IT & $\mathrm{C}, \mathrm{I}(\mathrm{U})$ & GI \\
\hline (30) & 10 & $70-100$ & 3050 & $1.210^{9}$ & M ID & $\mathrm{X}, \mathrm{H}$ & IT & $\mathrm{C}$ & $\mathrm{GI}$ \\
\hline (31) & Other & 60 & ND & $310^{9}$ & M ID & $x, 0$ & IT & I (Fluo) & $\mathrm{GI}$ \\
\hline (32) & 10 & 22 & 1497 & $4.310^{9}$ & M IC & $A, O$ & $\begin{array}{l}\text { IT and } \\
\text { IV }\end{array}$ & C & GI \\
\hline (33) & 10 & 16 & 360 & $1.810^{9}$ & M ID & $\mathrm{X}, \mathrm{H}$ & IT & $C$ & GI \\
\hline (34) & Other & $30-50$ & ND & ND & M IC & $A, H$ & IT & $\mathrm{C}$ & $\mathrm{GI}$ \\
\hline (35) & IC & 20 & 4850 & $1.0510^{9}$ & M ID & $\mathrm{X}, \mathrm{H}$ & IT & ND & $\mathrm{CR}$ \\
\hline (36) & IC & 30 & ND & ND & M ID & $\mathrm{X}, \mathrm{H}$ & IT & ND & CR \\
\hline (37) & 10 & 22 & 264 & $4.310^{9}$ & M ID & $\mathrm{X}, \mathrm{H}$ & IT & $\mathrm{C}$ & $\mathrm{GI}$ \\
\hline (38) & 10 & 10 & 76 & ND & M ID & $\mathrm{X}, \mathrm{H}$ & IT & $C$ & $\mathrm{GI}$ \\
\hline (39) & 10 & ND & ND & $5.910^{9}$ & M ID & $\mathrm{X}, \mathrm{H}$ & IT & C & $\mathrm{GI}$ \\
\hline (40) & 10 & ND & ND & $2.310^{9}$ & M ID & $x, 0$ & $\mathrm{INH}$ & I (Fluo) & $\mathrm{GI}$ \\
\hline (41) & 10 & 4 & 567 & $1010^{9}$ & M ID & $X, H$ & IT & $C, I(U)$ & $\mathrm{GI}$ \\
\hline (42) & IC & 30 & 76 & $5.910^{9}$ & M ID & $X, H$ & IT & $\mathrm{I}(\mathrm{CT})$ & CR \\
\hline
\end{tabular}

Table 12.1. Relevant data from all the papers revised. IO (Iron Oxides), IC (Iron Compounds), ND (Not Described), NP (Not particles), H CT (Human Clinical Trials), M IC (Mice 
ImmunoCompetent), M ID (Mice ImmunoDeficient), Rab IC (Rabbits ImmunoCompetent), Rat IC (Rat ImmunoCompetent), A (Allograft), X (Xenograft), O (Orthotopic), H (Heteretopic), IT (IntraTumoral), IV (IntraVenous), IA (IntraArterial), INH (Inhalation), C (Caliper), I (Imaging), U (Ultrasounds), MRI (Magnetic Resonance Imaging), Fluo (Fluorescence), CT (Computated Tomography), SR (Survival Rate), GI (Growth Inhibition), CR (Complete Regression).

\section{Nanoparticle design for MH in vivo application}

One can theoretically design and synthetize nanoparticles with optimal heating properties when measured in the laboratory (see Chapters 1 and 2). However, some limitations to the optimum nanoparticle design regarding their composition, size, and agglomeration start to arise when we reach in vivo applications. Moreover, in order to go one step further and envisage reaching the clinical practice, other factors start gaining importance such as the possibility for largescale production. In this section, all the implications on the nanoparticle design when focusing on $\mathrm{MH}$ are discussed in more detail.

\subsection{Nanoparticle composition}

MNP with different chemical composition have been prepared during the past years to evaluate their heating efficiency for hyperthermia purposes in aqueous suspensions, including materials such as cobalt ferrites (43), copper-nickel alloys (44) or copper-nickel (45) nanoparticles. However, when it comes to in vivo applications, toxicity issues arise and not all the chemical compositions are recommended given their potential toxicity. This is the main reason why the most commonly studied materials for in vivo magnetic hyperthermia treatments are iron oxides (46). In fact, in our literature review of in vivo pre-clinical hyperthermia treatments described in Table 1, we have found that the core composition of $\approx 68 \%$ of the materials tested was iron oxides (either magnetite $\left(\mathrm{Fe}_{3} \mathrm{O}_{4}\right)$ or maghemite $\left.\left(\mathrm{y}-\mathrm{Fe}_{2} \mathrm{O}_{3}\right)\right)$; most of the remaining compounds contained iron in their composition (including metallic iron, molecular iron compounds, doped ferrites or alloys). Only one paper used a completely different material $\left(\mathrm{LaSrMnO}_{3}\right.$ ) (See Fig. 12.2) (34).

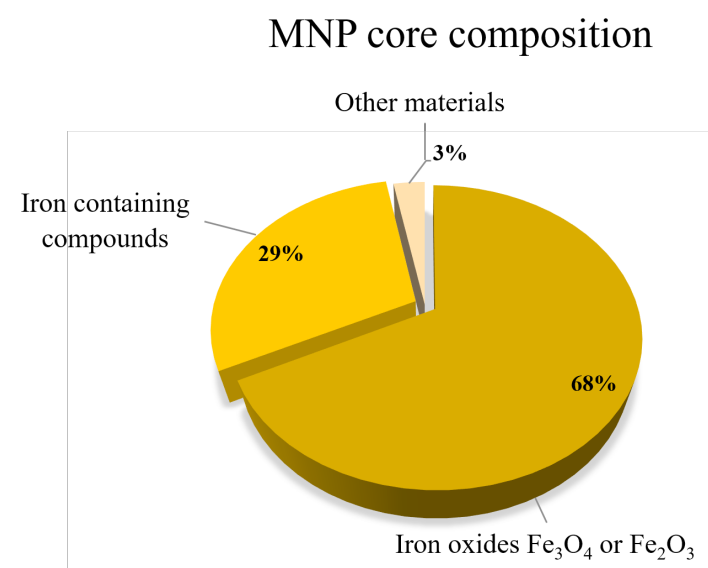


Fig. 12.2. Analysis of the MNP core composition used in our review of in vivo hyperthermia studies (Table 12.1).

The main advantage of iron oxides is the existence in the body of metabolic mechanisms to manage iron atoms, being able to store and transfer iron released from the nanoparticles during their degradation process (47). In fact, iron is such an important element in the treatment of widespread diseases (e.g. iron deficiency anemia among others) that several iron supplement formulations include iron oxyhydroxides (ferrihydrite or akaganeite) nanoparticles as part of their composition (48). It is less frequent however, to use iron oxide (magnetite or maghemite) nanoparticles as iron supplements (49). Iron oxides have also been approved by the Food and Drug Administration (FDA) of the USA as contrast agents for magnetic resonance imaging (MRI) (50), though most of the products have been discontinued since their approval (51).

\subsection{Nanoparticle size}

It is known that, in case of intravenous administration, the size of nanoparticles has a strong impact on their biodistribution. Nanoparticles with sizes below 5 $\mathrm{nm}$ are easily eliminated through the kidneys and those with sizes above 100 $\mathrm{nm}$ are rapidly cleared by macrophages and transported to the liver (52). These clearance routes limit the blood circulation time and, as a consequence, the possibility of reaching the targeting site in a concentration high enough for the clinical application. Therefore, for a better in vivo performance, nanoparticles should have sizes in the range between 5 and $100 \mathrm{~nm}$. This is the case of all the materials that have been recently reported in the literature. When looking in more detail, the largest amount (47\%) of nanoparticles used for the selected pre-clinical studies have sizes between 10 and $20 \mathrm{~nm}$ (see Fig. 12.3). This data have to be taken cautiously, as around $15 \%$ of the works do not indicate particle core sizes. It has also to be mentioned that most of the reported materials have a spherical shape, with only two examples of significantly different shapes (rods (12) and rings (30)). 


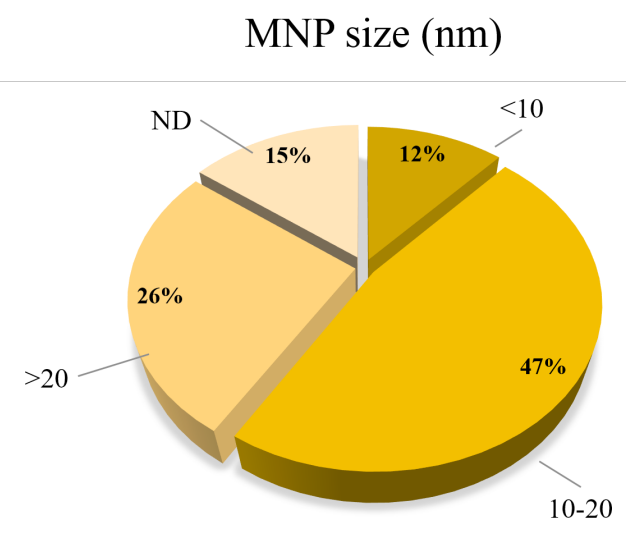

Fig. 12.3. Analysis of the MNP core size used in our review of in vivo hyperthermia studies (Table 12.1). (ND = Not described).

\subsection{Nanoparticle agglomeration}

Another limiting factor is nanoparticle agglomeration. The different colloidal properties that these materials present in aqueous suspension or in biological fluids may result in a strong agglomeration of nanoparticles in vivo. This has several consequences. On one hand, it may generate adverse effects during intravenous administration, if agglomerates are big enough to obstruct blood vessels. On the other hand, agglomeration has a strong impact on the magnetic properties of the materials, subsequently altering their heating performance. Unfortunately, these factors are often not evaluated in research manuscripts dealing with in vivo experiments.

\subsection{Nanoparticle synthesis route}

All these limiting factors, required for in vivo applications and described above, may point to a specific nanoparticle design. However, achieving the desired MNP through large-scale production is still a big challenge depending on the synthesis route.

Several synthesis routes are routinely followed to produce iron oxide MNP (see Chapter 2 for more details). Each approach presents its corresponding advantages and drawbacks, resulting in a compendium of procedures that allow the preparation of a wide diversity of materials with varying size, shape, and aggregation degree. Briefly, organic decomposition synthesis routes provide highly monodisperse nanoparticles (53) and polyol mediated synthesis allows the production of multicore structures controlling the final morphology of the particles (54). However, these two synthesis routes require high temperatures and the use of organic solvents. Aqueous protocols like the co-precipitation (55) and the hydrothermal aqueous routes (56) lead in general to aggregated 
nanoparticles with broader size distributions and not so well-defined morphology. However, these routes are low-cost, easy to scale-up and use non-toxic reagents.

From our literature review, regarding those studies using iron oxide nanoparticles, half of them provided a description of the preparation route of the nanoparticles (Fig. 12.4). The most common synthetic route for homemade particles was thermal decomposition (seven manuscripts), followed by coprecipitation (three manuscripts) and finally by polyol synthesis (one manuscript). Commercially available particles accounted for $18 \%$ of the studies analyzed (four manuscripts). Sadly, a significant percentage of the works (seven manuscripts, $32 \%$ ) did not provide enough information of the synthetic route of the magnetic cores, even without giving references to previous works describing the material. This is a great inconvenience for the progress of knowledge, as the systematic lack of details prevents an easier comparison of the results.

\section{MNP synthesis route}

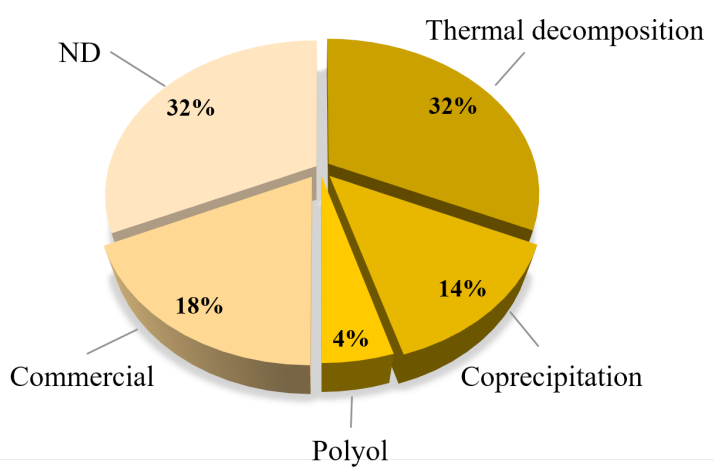

Fig. 12.4. Analysis of the MNP synthesis route used in our review of in vivo hyperthermia studies (Table 12.1). (ND = Not described).

The transference to the industry of all the knowledge acquired in the nanoparticle design is complex. The production of large amounts of some kind of materials is not straightforward due to scale-up difficulties resulting, in products not viable economically, and so it is with MNP. Even though it may seem that thermal decomposition is the preferred synthetic route for the obtention of iron oxide MNP for in vivo hyperthermia applications, it has to be pointed out that their production at large-scale (and under good manufacturing practices) is much more complex than that of the aqueous routes. When thinking on a clinical application, researchers should take into account the real possibilities of producing a given material at large-scale. In such case, aqueous routes should therefore be prioritized. Nevertheless, it should also be taken into account that scaling up processes are usually associated with a decrease in the 
homogeneity of the final product, and that it will be difficult to obtain exactly the same material for commercial purposes as the one obtained in smaller batches in research laboratories.

\subsection{Nanoparticle sterility}

Nanoparticles should be sterile for in vivo administration, to avoid the introduction of pathogens that may provoke undesirable biological effects in the animals and difficulties in achieving reproducible and consistent results.

In pre-clinical trials using MNP for biomedical applications, before the in vivo administration, magnetic nanoparticles are generally sterilized by filtration through small pores (e.g. $0.2 \mu \mathrm{m}$ ) that allows the removal of bacteria that may be present in the suspension. This procedure is sometimes complicated in case of agglomerated nanoparticles with bigger hydrodynamic diameters. This procedure was reported in three of the papers analyzed in our literature review $(13,14,38)$. Some of the manuscripts describe the use of sterile MNP although the sterilization process is not clearly detailed (20), and some others precipitate the MNP and just redisperse them in sterilized water $(22,27)$. This last procedure is not the most appropriated one to achieve a complete sterilization of the material. Unfortunately, the vast majority of the manuscripts revised for this chapter do not provide specific information on the MNP sterilization processes. If MNP have not been sterilized, this may have consequences on the animal response to the treatment affecting the interpretation of the response to the therapy and making more difficult to get reproducible results.

It also has to be taken into account that filtration does not eliminate endotoxins, which are bacterially derived molecules that may be a source of variability for in vivo studies $(57,58)$. The analysis of the presence of endotoxins is generally neglected in the literature, and the use of synthetic protocols to avoid common endotoxin contamination should be implemented, such as using high purity water and depyrogenated labware, handling of containers with clean gloves and testing reagents for endotoxin presence before their use. Future standardization protocols for in vivo hyperthermia studies should include the evaluation of the presence of endotoxins as a routine technique to evaluate the sterility of the materials prepared before in vivo applications.

\subsection{Nanoparticle heating properties}

MNP heating properties under an AMF are generally described by the specific absorption rate. SAR values are widely used to compare the heating capacity of different magnetic particles, although this extrinsic parameter depends on external factors such as the AMF amplitude and frequency, and the MNP concentration. An alternative approach is the determination of the Intrinsic Loss 
Power (ILP) and its determination has been addressed in more depth in Chapter 10.

In our literature review, SAR values of the suspensions of MNP, most of them in aqueous solutions, were provided in $\approx 60 \%$ of the manuscripts, with values ranging from 3 to $4850 \mathrm{~W} \mathrm{~g}^{-1}$. These values have limited interest, as the conditions in which they were measured are completely different from one work to another; in addition, the local concentration in the tissue samples is unknown. This is a consequence of the lack of real-time and accessible characterization techniques that provide a fast, cheap and reliable quantification of the MNP in the tumor.

\section{Magnetic hyperthermia conditions used in vivo}

In this section, we are going to review and discuss some parameters of the $\mathrm{MH}$ application, such as the values of the magnetic field amplitude and the frequency used and the number of repetitions and duration of the AMF exposure from the studies detailed in Table 12.1.

\subsection{Equipment}

In Chapter 5, a detailed description of the instrumentation for SAR determination and application of magnetic hyperthermia in vitro and in vivo is given. Here, our aim was to compare the equipment used in the literature manuscripts reported in Table 12.1 to try to establish any trend on the instrumentation being used for in vivo applications. However, we have found that there is no clear trend on the equipment used, as the vast majority of the research papers reported homemade equipment.

The heterogeneity of instrumentation used for hyperthermia applications leads to a plethora of field amplitudes and frequencies resulting in a very broad range of conditions used for the in vivo experiments. Each of the described instruments produces an almost unique alternating current (AC) magnetic field, in terms of frequency and amplitude of the magnetic field. Therefore, it is almost impossible to compare the results among different studies. The frequency of the AC magnetic field ranged between 0.02 and $1300 \mathrm{kHz}$, being the most common ones $(\approx 62 \%$ ) located in the range between 360 and $650 \mathrm{kHz}$ (see Fig. 12.5). In a similar way, field amplitudes used were in the range between 0.6 and 150 $\mathrm{kA} / \mathrm{m}$, being the most common ones $(\approx 47 \%)$ in the range between 9 and 20 $\mathrm{kA} / \mathrm{m}$ (see Fig. 3.1.1). 


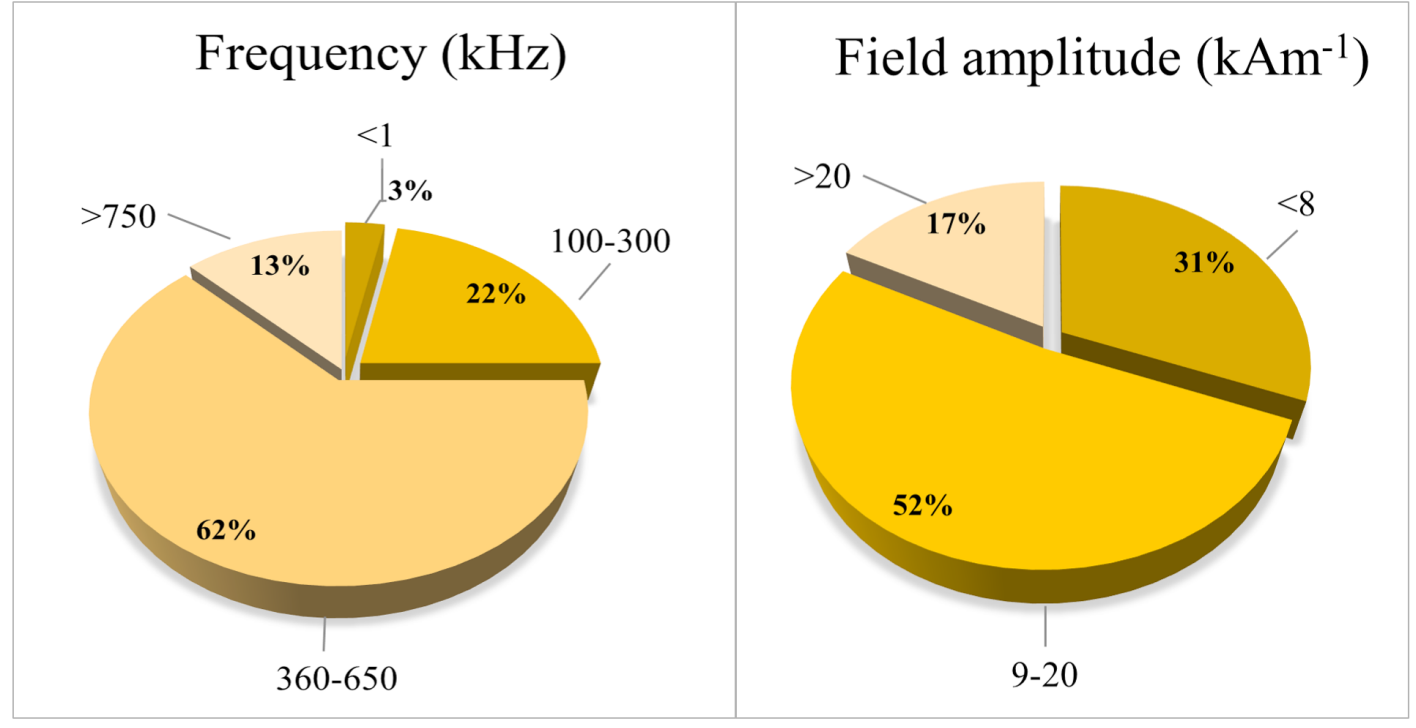

Fig. 12.5. Analysis of the frequency and field amplitude used in the in vivo hyperthermia studies from Table 12.1.

Given the broad spectra of AMF conditions, the comparison of the results from the different studies is a complex task, especially taking into account that for a given frequency and amplitude of the applied magnetic field, the heating performance of magnetic particles depends on their size and composition (59). In such scenario not all the nanoparticles may have the same behavior in every instrument.

Moreover, even if the particle size and composition were optimized for a given set of experimental frequency and amplitude of the AC magnetic field conditions, another factor, the nanoparticle concentration should be also taken into account, to achieve an optimal design (60).

Given that so many different parameters have such a complex influence on the final clinical performance of MNP during in vivo hyperthermia treatments, it is extremely difficult to design the optimum material for $\mathrm{MH}$ purposes. Therefore, even if MNP and field conditions were designed together to achieve the most efficient combination for heat generation, this final combination should also take into account the sample dose needed for the clinical application to achieve the maximum effectiveness.

\subsection{Biological safety of the AMF}

When an AMF is applied to tissues not only the therapeutically useful heating of MNP takes place but also the tissue itself begins to non-selectively heat due to 
the generation of eddy currents. There exists an open deliberation about the most convenient values for amplitude and frequency parameters, concerning biological safety, since Atkinson et al. published in 1984 their very well-known paper where they established the range of usable frequencies in MH (61) (see Chapter 1 for more details). In that manuscript, the authors concluded that the value of the product $\mathrm{H}^{*} \mathrm{f}$ should not exceed $4.8510^{8} \mathrm{Am}^{-1} \mathrm{~s}^{-1}$. This was the highest well-tolerated condition that people receiving the treatment for more than one hour could withstand without major discomfort. They showed that the rate of heat produced per tissue is directly proportional to the square of $\mathrm{H}^{*} \mathrm{f}$ product and also to the square of the distance from the coil center. So, the smaller the radius of the region to be treated, the higher $\mathrm{H}^{\star} \mathrm{f}$ values could be applied. As all the experiments were carried out with a coil designed to treat the thorax (about $30 \mathrm{~cm}$ ), authors opened the possibility to use higher $\mathrm{H}^{\star} \mathrm{f}$ limits for smaller applicators.

Hergt and Dutz established a new and weaker criterion for this limit for smaller coils and relating to the seriousness of the illness, dictating that the limit for the $\mathrm{H}^{* \mathrm{f}}$ product was $510^{9} \mathrm{Am}^{-1} \mathrm{~s}^{-1}$. Thus, these authors made theoretical calculations to maximize the SAR of MNP when exposed to AMF respecting the biological safety parameters (62).

If we analyze the AMF conditions used in the pre-clinical studies from Table 12.1 (Fig. 12.6) we can see that none of the papers we have revised has used values of $\mathrm{H}$ and $\mathrm{f}$ that result in $a \mathrm{H}^{*} \mathrm{f}$ value below the limit proposed by Atkinson, $4.8510^{8} \mathrm{Am}^{-1} \mathrm{~s}^{-1}$. Half of the papers used AMF conditions between $4.8510^{8} \mathrm{Am}^{-}$ ${ }^{1} \mathrm{~s}^{-1}$ and $510^{9} \mathrm{Am}^{-1} \mathrm{~s}^{-1}$, so above the Atkinson limit but below the limit proposed by Hergt and Duzt. This range seems to be currently the most convenient one, at least until new studies about the biological safety of the AMF are carried out. However, there are $35 \%$ of the studies that used $\mathrm{H}$ and $\mathrm{f}$ parameters resulting in $\mathrm{H}^{*} \mathrm{f}$ values above the biological safety limit.

The highest condition of the $H^{*} f$ product we have found is the article published by Guo et al. $\left(10^{10} \mathrm{Am}^{-1} \mathrm{~s}^{-1}\right)$, doubling the convenient limit. The application time used in this work was five minutes and the temperature of the surroundings was not measured. They combined $\mathrm{MH}$ with laser irradiation as some studies have demonstrated that $\mathrm{Fe}_{3} \mathrm{O}_{4}$ nanoparticles are able to exhibit strong photothermal effects when exposed to NIR laser irradiation (63). So, after combining MH with laser irradiation and chemotherapy, the results obtained were good showing tumor growth inhibition after two weeks post-treatment (41).

Not less important is to mention that some published studies $(15 \%)$ do not describe the conditions of the AMF used, so it is quite difficult to correlate and position them within the research field of $\mathrm{MH}$. Besides, with some papers, we have had difficulties to find the AMF conditions used, as it was necessary to 
track back a large number of previously published works. Our particular point of view in this respect is that when publishing a research work in this field some data need to be always mentioned, in particular, AMF conditions should always be provided.

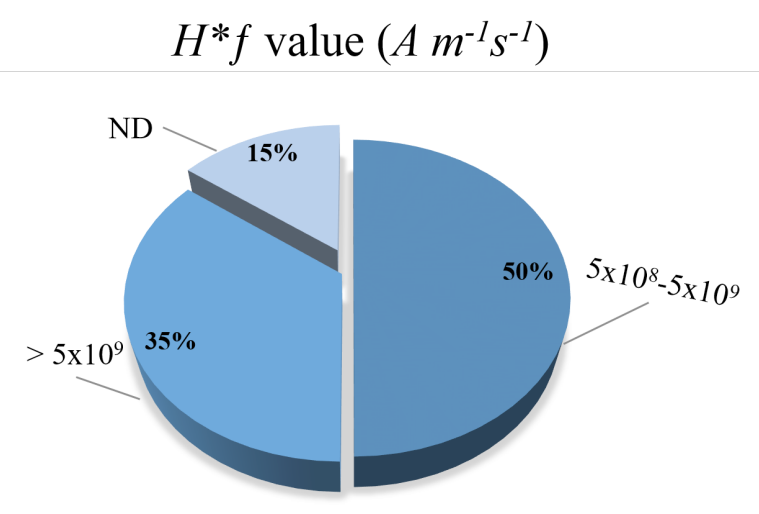

Fig. 12.6. Representation of the $\mathrm{H}^{*} \mathrm{f}$ values used. Data taken from all the papers detailed in Table 12.1.

\subsection{Exposure to the AMF}

The methodology followed for the application of the $\mathrm{MH}$ treatments varies between published works not only in the AMF conditions used but also in other aspects, such as the duration of the AMF exposure and the number of consecutive applications.

As shown in Fig. 12.7 the duration of the AMF exposure in most of the reviewed studies $(41 \%)$ is between 10 and 30 minutes. In $35 \%$ of the studies the exposure time is higher, between 30 and 60 minutes. Only $18 \%$ of the papers reveal exposure times below 10 minutes. Is it quite difficult to elucidate in advance which is the best exposure time to obtain the best antitumor effect. We tend to think that the longer exposure time, the stronger the effect. But there exist some limitations that have to be considered in this aspect, for example, the technical limitations of the devices, as in some of them the AMF application cannot exceed certain duration. Also, the kind of dispositive used to maintain the temperature of the animals during the treatment is important. Some of the devices had a 3D temperature regulation system that prevented the animals from suffering hypothermia in a much better way than systems consisting of a hot surface where the animals are just laid down.

The number of repetitions of the AMF exposure and the intervals between them also differ between studies. As presented in Fig 12.7, 23\% of the studies applied $\mathrm{MH}$ just once, $12 \%$ applied $\mathrm{MH}$ twice, $21 \%$ of the studies performed the 
$\mathrm{MH}$ three times, $9 \%$ of the papers performed four $\mathrm{MH}$ exposures and $6 \%$ of the studies reviewed applied the magnetic field five or more consecutive times. Again, it is hard to conclude which is the best treatment option, as the degradation of the MNP in the tissues should be taken into account. In particular, MNP degradation will strongly depend on their composition and coating (47). It is now well known that the exposure of iron oxide MNP to an acidic medium could dramatically affect their heating capability. As an example, experimental studies have shown how the heating capacity of iron oxide nanoflowers (with polyols as organic protecting shell) decreased $70 \%$ and $99 \%$ after 6 and 23 days respectively, under acidic media exposure (64). It is quite obvious that the exposure of the animal to the AMF at certain time points after the MNP injection if they have been degraded or removed from the target tissue will be ineffective.

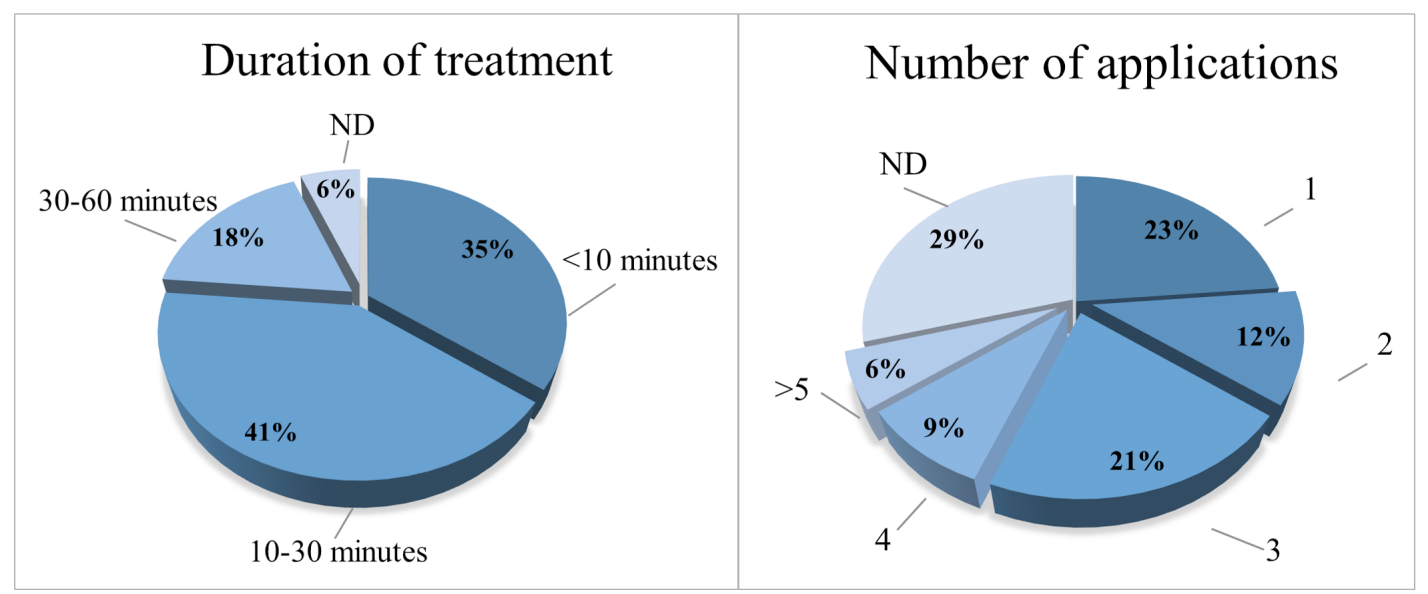

Fig. 12.7. Representation of the duration of the AMF exposure and the number of consecutive repetitions of the treatment. Data taken from the papers detailed in Table 12.1.

From these results, it can be extracted that the most common protocol for this kind of experiments uses between one or three AMF applications (in alternating days) with a duration length between 10 and 30 minutes. It is noteworthy again the number of studies that do not provide enough details on the methodology used, thus hindering the standardization of in vivo $\mathrm{MH}$ experiments.

\section{Animal models and biological effects}

In this section, animal models, tumor models and MNP administration routes are reviewed in the frame of the in vivo $\mathrm{MH}$ experiments selected and detailed in Table 12.1. Also, we discuss the biological effects that are analyzed after the $\mathrm{MH}$ treatment and the techniques currently used for their study.

\subsection{Animal models}


Evaluation of the $\mathrm{MH}$ treatment effectiveness in small animal models is a necessary pre-clinical step before reaching human studies. Although several animal models exist, murine models are by far the most extended ones for pre-clinical trials. This is also the case for MH studies. The majority of works published until now (85\% of the reviewed papers) used mice as animal model for in vivo hyperthermia studies (Fig.12.8). Rats and rabbits have been used scarcely. The main advantages of mice relate to their small size, easy handling, fast reproductive cycles and their fully known genome.

Murine models can be further classified into two types, depending on the role of the immune system in the animals: immunocompetent and immunodeficient models. Immunocompetent mice are the most commonly used model in oncoimmunology for the inoculation of histocompatible cancer cell lines (65). However, in our literature review, they represent only a small proportion of the murine models used for in vivo hyperthermia studies (12\%). In particular, the four studies that have used immunocompetent models have worked with C57BI/6j mice, a commonly used strain for in vivo studies. Immunodeficient models are more frequently used (88\% from the papers revised) (Fig.12.8) in our literature review. The main reason for this trend is that these mice can accept and develop normal and malignant grafts both from donors of the same species (allograft) as well as from different species (xenografts - usually from human origin). The main disadvantage of these animal models is the lack of a complete immunologic response. Although not deeply studied yet, there is some scientific evidence about the synergy between different thermal treatments (photothermal, $\mathrm{MH}$, radiofrequency) and immunotherapy (66). So, the lack of immune system, which usually plays a key role to combat diseases, could negatively influence the treatments underestimating their real effect. There is also a study where the effectiveness of the $\mathrm{MH}$ was enhanced due to the administration of immunestimulators such as IL-2 (Interleukine-2) and GM-CSF (Granulocyte and Monocytes colony-stimulating factor), revealing the great importance of the immune response in the final effectiveness of the treatment (67). 


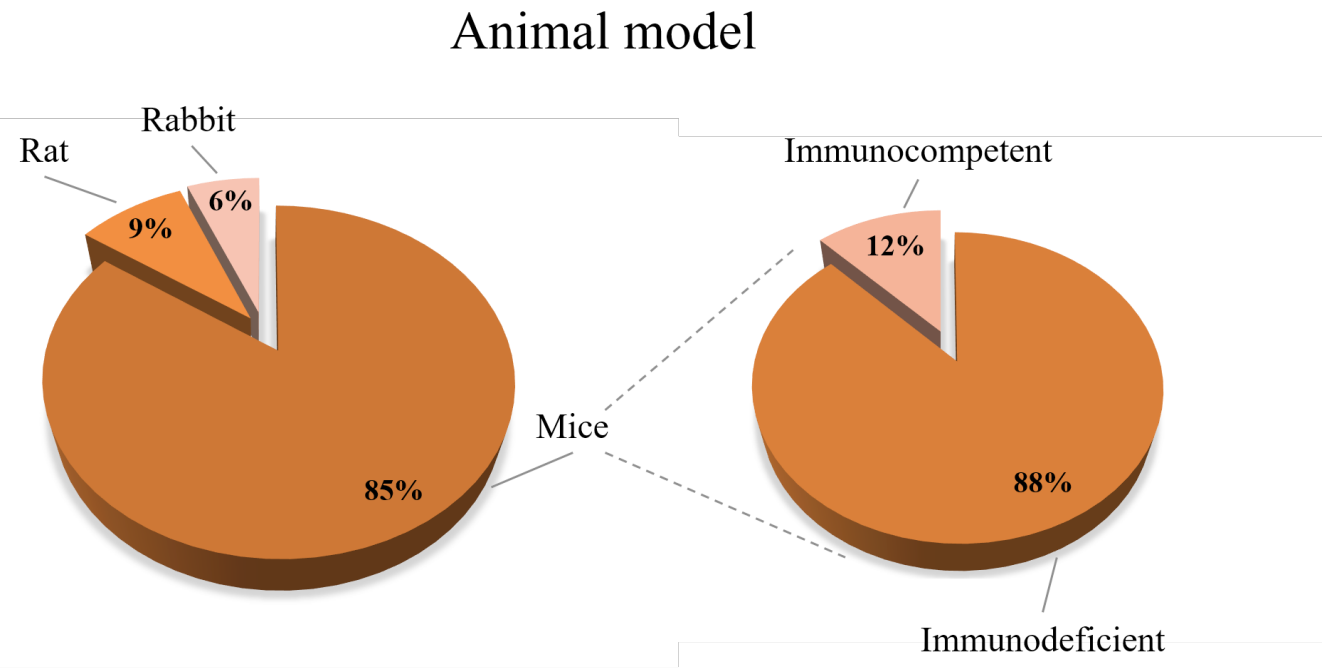

Fig. 12.8. Schematic representation of the principal animal models used for in vivo hyperthermia studies. Data taken from all the papers detailed in Table 12.1.

Several immunodeficient models, which are commercially available and that have been used in $\mathrm{MH}$ studies, are described next, detailing their specific advantages. One of the most commonly used immunodeficient models for hyperthermia studies are nude (nu) mice, named after their hairless phenotype. These mice are characterized by a severe reduction in the number of functional $T$ cells as a result of having a dysfunctional and rudimentary thymus that remains small and cystic throughout the animals' life. As a consequence of this, failures in the maturation of their $T$ cells occur. However, they present a response by the innate immune system and also humoral response. The homozygote model is the most widely distributed commercially and is available in various genetic backgrounds, consanguineous and non-consanguineous. The nude BALB/c type is the most reported in the literature, probably due to the fact that they are generally easy to breed and present minimal weight variations between females and males. Other variants used to a lesser extent with nude mutation in Foxn $1^{\text {nu }}$ (forkhead box N1) are Athymic Nude-nu from Charles River (NCR-Foxn1nu) or Athymic nude from Naval Medical Research Institute (NMRI). The fact that nude mice have scarce normal hair follicles makes them an attractive model for evaluating the growth of subcutaneously established tumors since epilation is not necessary to follow-up tumor growth. Another advantage of this hairless model is that it allows following labeled tumor cells by fluorescence imaging or by bioluminescence acquiring whole body images of the animals. 
In general, these kind of nude mice are good models to establish rapidly growing human tumor cell lines. However, as they maintain a robust humoral and natural killer cell (NK) responses, nude mice are not adequate for bloodborne tumor lines such as leukemias or lymphomas. Moreover, they are also not adequate for human primary lines or heterogenetic tumor fragments. For this reason, another type of immunodeficient mice model used also in vivo $\mathrm{MH}$ experiments is the SCID (severe combined immunodeficiency) mouse model. The homozygous scid/scid (Prkdc ${ }^{\text {scid }}$, catalytic subunit of a DNAdependent protein kinase) mice are phenotypically normal and have very low levels, or directly lack serum immunoglobulins. These animals have an inability to generate an immune response, both humoral and cellular (absence of mature $\mathrm{T}$ and $\mathrm{B}$ cells). This model presents superior success rate than the nude mice in human tumor transplants(52). However, SCID mice mutations do not affect the myeloid and erythroid cell lines and therefore, these models exhibit normal levels of NK activity.

\subsection{Tumor model and relationship with the administration routes}

In general, one of the major problems observed when performing in vivo experiments using nanoparticles is the low amount of material that reaches the desired location after systemic administration. In fact, there is a recent study that, after revising more than one hundred papers, concluded that less than $1 \%$ of the intravenously administered dose arrives to the tumor (68). This presents a real problem for biomedical applications that require a high amount of nanoparticles in the target tissue, as it is the case of $\mathrm{MH}$. Because of this, the majority of the published studies $(80 \%)$ use the direct injection of the magnetic material at the tumor site as main route, therefore ensuring that the highest possible MNP content remains in this site (Fig. 12.9). However, this type of administration has the disadvantage that requires the establishment of subcutaneous tumors. Heterotopic tumors are the ones located outside the equivalent organ where the tumors should be grown, as the subcutaneous implants. The major drawback is that the tumor microenvironment in the subcutaneous model is very different from that of the primary cell or tissue type that generates the tumor. Precisely these interactions between the host envelope and tumor tissue strongly affect tumor cell proliferation, levels of growth factors and nutrients, both during tumor angiogenesis and in its metastatic behavior. All these reasons constitute the major limitations of subcutaneous tumor models. On the contrary, they are very easy to handle. The accessibility of subcutaneous tumors is a great advantage to monitor tumor growth progression and for the assessment of the direct effects of $\mathrm{MH}$ in its regression. For these reasons, this kind of tumor model is the most commonly used ( $74 \%$ reviewed papers) 
(Fig. 12.10).

Administration routes

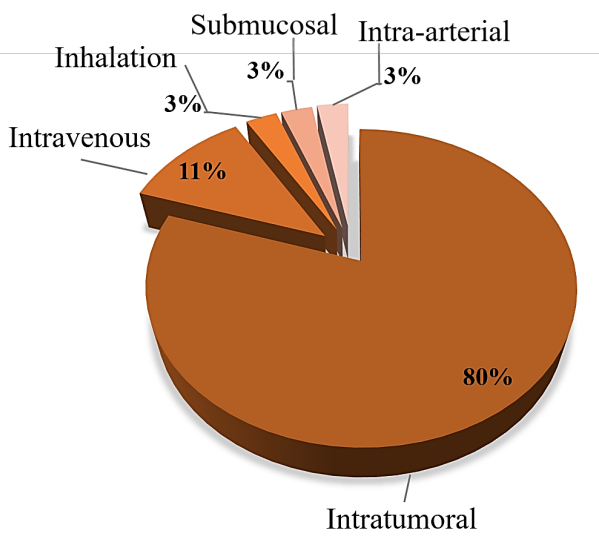

Fig. 12.9. Schematic representation of the administration via of magnetic material for in vivo hyperthermia studies.

Orthotopic tumors are the ones implanted or generated into the equivalent organ from which the cancer originated. This type of implantation results in the correct microenvironment, which may more closely mimic the natural tumorigenesis in humans, as opposite to heterotopic models. These models are considered more clinically relevant and are better predictive models of drug effectiveness than standard subcutaneous models.

\section{Tumor model}

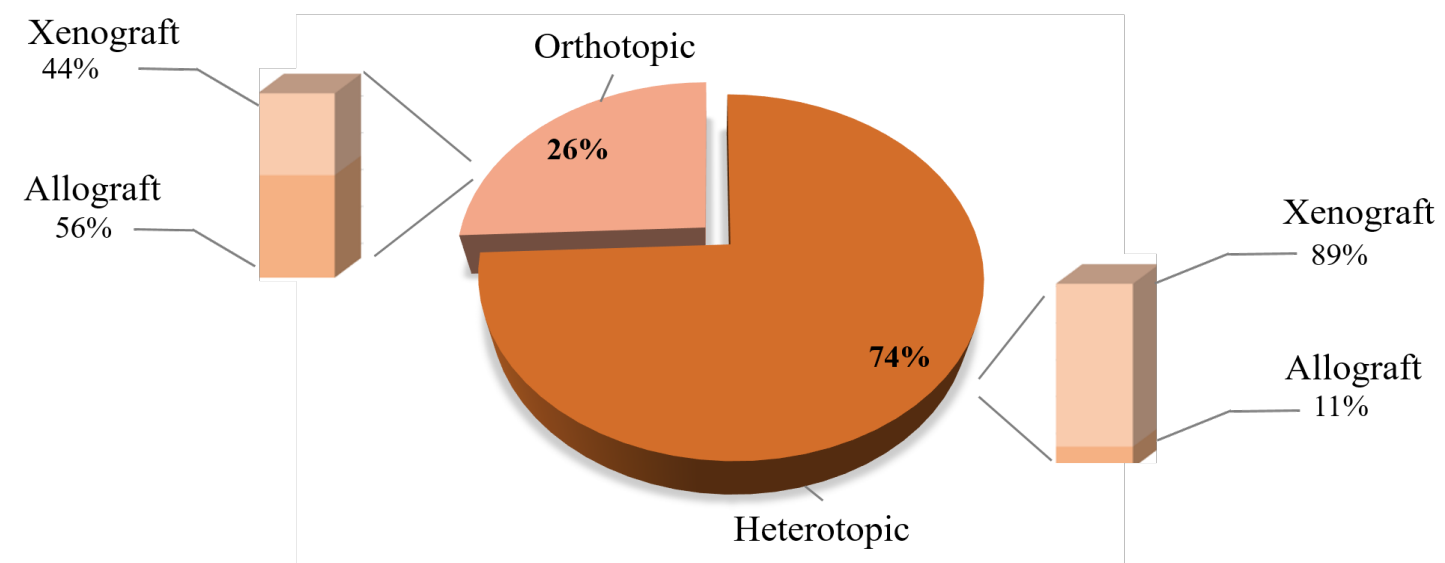

Fig. 12.10. Schematic representation of the percentage of the different tumor models used for in vivo hyperthermia studies.

However, although orthotopic tumor models are considered ideal for 
evaluating the real impact of therapy, they have not been established yet as standard models (Fig. 12.10). They require more complex imaging methods to follow their growth, and they also need more technical expertise and, in general, it can be said that it is a labor-intensive task. But one of the main reasons for the preference of the subcutaneous model over orthotopic tumors is the fact that currently this is the only way to ensure that most of the nanoparticles remain within the tumor for long periods of time. In the case of intratumoral injection of MNP, it has been shown that around $89 \%$ of the injected MNP get immobilized in the tumor tissue. MNP are not only retained in the tumor interstitium, but they are also tightly packed inside endosomes of tumor cells and macrophages associated to the tumor. Besides, even after $\mathrm{MH}$ sessions using a combination of high field amplitude and frequency (25 $\mathrm{kAm}^{-1}$ and $400 \mathrm{kHz}$ ), they remained unaltered as homogeneous spots. However, it is noteworthy to highlight that not only their distribution within the tumor but also the time window where their heating capabilities are preserved before undergoing degradation must be determined for each type of MNP (8). Published studies of systemic administration require bigger doses to ensure that the necessary concentration of MNP to produce a biological effect reaches the tumor site (68). One alternative to solve this problem is the intraarterial administration of MNP (69). This administration route allows a more selective and at the same time more widely spread distribution of the hyperthermic agent. The main disadvantages are the technical difficulties and the possible escape of the therapeutic material to the general circulation, with its consequent toxic effects.

As can be seen in Fig. 12.10 when the tumor model is located subcutaneously (heterotopic) the majority of the studies, $89 \%$, were carried out using immunodeficient mice where human tumors were implanted (xenograft tumor models). These models are a very easy and fast approach that renders an accessible tumor being the easiest in vivo models for preclinical human tumors studies. However, at the same time, they do not provide the appropriate environment and lack the complete effect of the immune system. Only the $11 \%$ of studies with heterotopic tumors of our selection were made with murine tumor cells in immunocompetent animals where the advantage is the contribution of the immune response. However, the scenario is different when analysing the studies where an orthotopic tumor is used, where more than half of them consist of murine cells. As mentioned before, the technical experience needed for the development and follow up of these models is much higher, but a more realistic tumor environment and is mimicked. However, a part of the reviewed orthotopic tumors (44\%) were implanted in immunodeficient mice using human cells. It is true that in these cases the environment is much appropriate than in 
heterotopic tumors but there is a lack of immune system response.

Another aspect to take into account in the choice of the tumor model is the way the tumor is induced. Two main alternatives have been found in our literature research, either by injection of tumor cells or by surgical implantation of tumor fragments. Most of the studies reported used material from cell cultures for both heterotopic and orthotopic models. It should be noted that in the case of the latter some authors preferred to use fragments of tissue mainly in larger animal models such as rabbits and rats. These fragments (usually $1 \mathrm{~mm}^{3}$ ) were usually obtained from previous tumor growth in the same animal model in which it will be subsequently grafted. However, a major disadvantage of models utilizing intact tumor tissue is the intrinsically heterogeneous nature of the different zones within the same tumor. This makes very difficult to standardize and compare results between tumors from different subjects. An alternative to avoid this heterogeneity is to resuspend in balanced saline pieces of the tumor tissue obtained from the different subjects (with a concentration of 100 pieces per milliliter), and then, inject this mixture to the experimentation subject.

\subsection{Biological effects observed and techniques used}

There are several techniques to study the antitumor effect of the $\mathrm{MH}$ treatment but there are two that stand out because they are the most commonly used. These two techniques are: physical measurement of the tumor dimension with a caliper and the use of imaging techniques to follow the tumor growth.

There are some advantages and drawbacks that have to be taken into account when one should decide which technique is going to be used to follow the tumor growth. The direct measurement of the tumor with a caliper is a very simple, fast and easy way to estimate the volume also allowing the possibility to daily follow their growth. But it is true that it is not the most accurate methodology because, for example, inflammatory tissue and necrosis zones may lead to an overestimation of the tumor volume. Besides, the operator in charge of such task should always be the same to minimize errors. The accuracy of the measurements will depend on the kind of tumor implanted and the animal model used. The tumor shape could be different and therefore, assumptions made to calculate the final volume may not be reliable. In this sense, the type of skin, color and presence of hair could facilitate or restrain the measurements. Furthermore, different equations to calculate final volume exist, the following two being the most used ones:
1) $V=\frac{\pi}{6} a b c$
2) $V=\frac{1}{2} a b^{2}$ 
Eq. 1 is the volume of an ellipsoid where $a, b$ and $c$ are the three tumor dimensions (length, width, and height). Eq. 2 corresponds to a modified ellipsoid equation where two dimensions, tumor height and width, are considered to be the same. The most accurate formula is the first one because it takes into account the three dimensions, although using a caliper to measure the height of the implanted tumors is not always an easy task. Thus, the modified ellipsoid volume formula is easier for the calculations as it makes the assumption that the height of the tumors is the same as the width. However, it should be taken into account that this assumption is not always correct. Nevertheless, using this approximation to compare tumor volumes from animals from the same experiment can be considered as relevant.

Other types of techniques used to follow the tumor growth are imaging techniques. They are more accurate than measurements made with a caliper but they are quite costly and have also their own technical limitations. There are several imaging techniques such as: 1) Magnetic Resonance Imaging (MRI) which is based on the different relaxation times of the protons depending on their environment after being pulsed with a strong magnetic field in the range of radiofrequency, 2) Computated Tomography (CT) which detects different absorption of X-ray radiation between water, bone, fat and air, 3) Medical ultrasounds (diagnostic sonography) which is based on the differences in the time that takes for a pulse of sound to interact with a tissue and travel back to the probe depending on their different acoustic impedance and 4) bioluminescence and fluorescence imaging devices that detect the fluorescence or photons coming from the animal. This last technique requires having the tissue expressing a fluorophore protein or an enzyme that catalyzes bioluminescence reactions. The spatial resolution of all of these techniques is quite similar: MRI between 4-100 $\mu \mathrm{m}$, CT 12-50 $\mu \mathrm{m}$, Ultrasound $50 \mu \mathrm{m}$ and bioluminescence or fluorescence in vivo imaging also $50 \mu \mathrm{m}$ (70). However, MRI, CT and Ultrasound are independent of the tumor depth, while bioluminescence and fluorescence imaging can only be used for real-time imaging when the tumor is subcutaneous. The most popular ones used in the revised bibliography were MRI and fluorescence imaging.

When we analyze the selected papers (Fig. 12.11) we can see that more than half $(57 \%)$ used only a caliper to calculate the volume of the tumors, while $23 \%$ of the studies followed tumor development with imaging techniques, whereas $17 \%$ of the studies combined both a caliper and imaging. This last combination allows correlating the signals obtained by the imaging techniques (e.g. photon flux in fluorescence) with tumor volume estimated with the caliper. This combination may not be necessary if the imaging approach is able to provide $3 \mathrm{D}$ reconstructions of the tumor volume. 

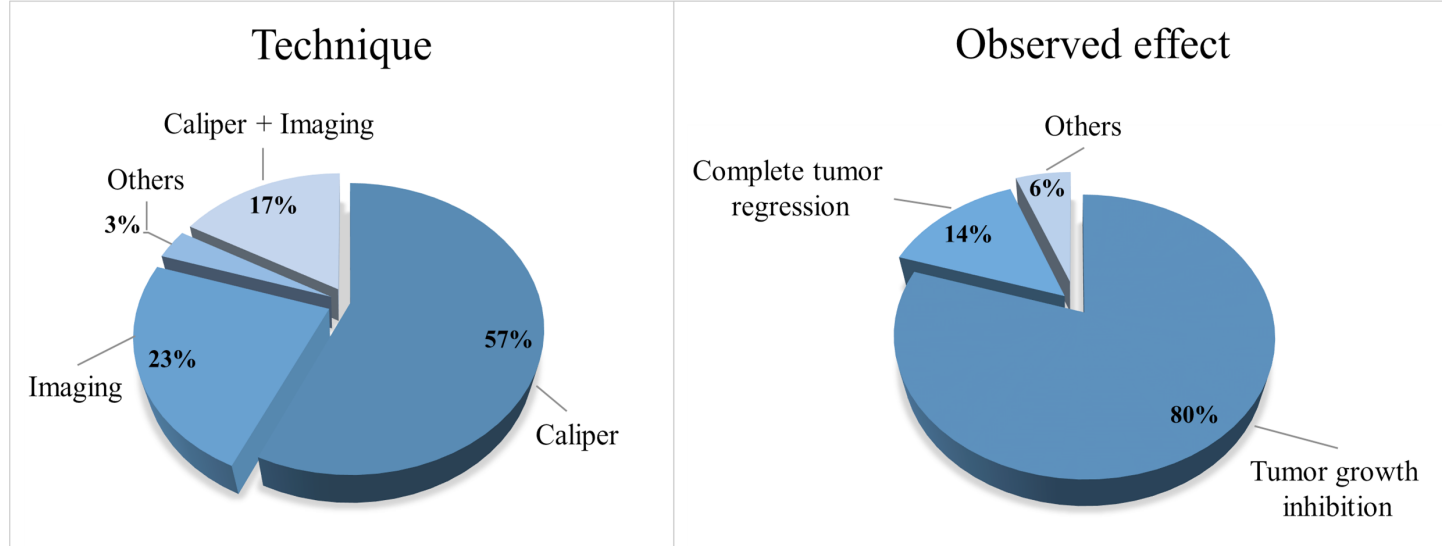

Fig. 12.11. Representation of the techniques used to analyze the biological effect of the treatment. Data taken from all the papers detailed in Table 12.1.

There is just one manuscript of the reviewed ones that used a different approach to determine tumor volume. It is the case of the study of the antitumor effect of the treatment described by Wang et al. that is based on the measurement of the achieved apoptosis rate by the analysis of the expression of Bax/Bcl-2 proteins, using terminal deoxynucleotidyl transferase dUTP nick end labeling (TUNEL) and Transmission Electron Microscopy (TEM) analysis (11).

In spite of the diverse treatments conditions and methodologies used to check the $\mathrm{MH}$ effectiveness, $80 \%$ of the revised works concluded that there is a tumor growth inhibition after the treatment. In addition, $14 \%$ of the cases reported a complete tumor regression, with no signs of tumor regrowth at least one month after ending the $\mathrm{MH}$ treatment $(36,42)$. However, tumor volume regressions are sometimes difficult to compare among different studies as different time points are analyzed. It is truth that in some cases the antitumor effect produces a very slight inhibition of the growth. That is why many recent studies are now focusing on the synergistic effect of this treatment with other therapies such as chemotherapy or radiotherapy.

One conclusion that could be extracted from this comparison is that the $\mathrm{MH}$ studies that have been made are successful as tumor growth inhibition is observed in most cases. However, it is worth mentioning that negative results are often not published, which could lead to an overestimation of the effectiveness of this treatment.

\section{Limitations and future challenges}


Right now, one of the major problems that the scientific community working on in vivo $\mathrm{MH}$ is facing is the difficulty to compare results from different laboratories, as little attention has been paid to the standardization of protocols. Also, in many cases, negative results are not being published.

Most of the manuscripts revised for this book chapter do not provide a complete description of the materials and methodology used. This is a consequence of the multitude of parameters that are involved in such complex animal experiments. However, the limited access to these details makes more complex the comparison between research works.

Part of these problems could be solved by a general use of commercial devices specifically designed for in vivo $\mathrm{MH}$ application, as this would imply a narrower range of AMF conditions. Moreover, it would also be ideal that each of the equipment was commercialized with a given type of MNP, specifically optimized for the field conditions of the applicator. In this way, optimal heating properties would be achieved during the in vivo experiments.

The animal models used in the majority of the works revised consist of human cell lines subcutaneously implanted in immunodeficient mice. Although this kind of model is adequate as proof of concept of the treatment, more complex animal models involving the correct tumor environment and a complete immune response are needed for a better assessment of the treatment effectiveness in real conditions. This implies the use of more complex imaging techniques to follow the progress of the tumor growth after the treatment. In addition, an effort should be made on evaluating the $\mathrm{MH}$ treatment effectiveness in tumors with bad prognosis.

There are other two major issues that could also help to improve the $\mathrm{MH}$ treatments. Monitoring MNP localization using imaging techniques (e.g. MRI and CT imaging) $(71,72)$ before the treatment would allow the use of mathematical algorithms to design personalized $\mathrm{MH}$ application conditions by calculating the heat generation depending on the particle location within the tumor. Being able to measure the in situ temperature in a non-invasive way during the $\mathrm{MH}$ application would also help to understand the effectiveness of the treatment using different materials and conditions.

Despite the fact that this chapter has focused mainly on $\mathrm{MH}$, the general trend is to use this treatment in combination with currently established ones such as chemotherapy, radiotherapy, surgery or immunotherapy. Synergies among these treatments may significantly improve the final antitumor effect. 
Nevertheless, the main real future challenge would be to overcome the difficult gap between pre-clinical animal studies and the real clinical practice with humans.

\section{References}

1. Beik J, Abed Z, Ghoreishi FS, Hosseini-Nami S, Mehrzadi S, Shakeri-Zadeh A, et al. Nanotechnology in hyperthermia cancer therapy: From fundamental principles to advanced applications. Journal of Controlled Release. 2016;235:205-21.

2. Cervadoro A, Giverso C, Pande R, Sarangi S, Preziosi L, Wosik J, et al. Design maps for the hyperthermic treatment of tumors with superparamagnetic nanoparticles. Plos One. 2013;8(2):e57332.

3. Datta N, Krishnan S, Speiser D, Neufeld E, Kuster N, Bodis S, et al. Magnetic nanoparticle-induced hyperthermia with appropriate payloads: Paul Ehrlich's "magic (nano) bullet" for cancer theranostics? Cancer Treatment Reviews. 2016;50:217-27.

4. Jordan A, Scholz R, Wust P, Fähling H, Krause J, Wlodarczyk W, et al. Effects of magnetic fluid hyperthermia $(\mathrm{MFH})$ on $\mathrm{C} 3 \mathrm{H}$ mammary carcinoma in vivo. International Journal of Hyperthermia. 1997;13(6):587-605.

5. Yadollahpour A, Hosseini SA. Magnetic nanoparticle based hyperthermia: A review of the physiochemical properties and synthesis methods. International Journal of Pharmaceutical Research and Allied Sciences. 2016;5(2):242-6.

6. Salunkhe AB, Khot VM, Pawar S. Magnetic hyperthermia with magnetic nanoparticles: a status review. Current Topics in Medicinal Chemistry. 2014;14(5):57294.

7. Kafrouni L, Savadogo O. Recent progress on magnetic nanoparticles for magnetic hyperthermia. Progress in Biomaterials. 2016;5(3-4):147-60.

8. Perigo EA, Hemery G, Sandre O, Ortega D, Garaio E, Plazaola F, et al. Fundamentals and advances in magnetic hyperthermia. Applied Physics Reviews. 2015;2(4):041302.

9. Maier-Hauff K, Rothe R, Scholz R, Gneveckow U, Wust P, Thiesen B, et al. Intracranial thermotherapy using magnetic nanoparticles combined with external beam radiotherapy: results of a feasibility study on patients with glioblastoma multiforme. $\mathrm{J}$ Neurooncol. 2007;81(1):53-60.

10. Balivada S, Rachakatla RS, Wang H, Samarakoon TN, Dani RK, Pyle M, et al. $\mathrm{A} / \mathrm{C}$ magnetic hyperthermia of melanoma mediated by iron(0)/iron oxide core/shell magnetic nanoparticles: a mouse study. BMC Cancer. 2010;10(1):119.

11. Wang P, Xie X, Wang J, Shi Y, Shen N, Huang X. Ultra-small superparamagnetic iron oxide mediated magnetic hyperthermia in treatment of neck lymph node metastasis in rabbit pyriform sinus VX2 carcinoma. Tumor Biology. 2015;36(10):8035-40.

12. Rachakatla RS, Balivada S, Seo G-M, Myers CB, Wang H, Samarakoon TN, et al. Attenuation of Mouse Melanoma by A/C Magnetic Field after Delivery of BiMagnetic Nanoparticles by Neural Progenitor Cells. ACS Nano. 2010;4(12):7093-104.

13. Kossatz S, Ludwig R, Dähring H, Ettelt V, Rimkus G, Marciello M, et al. High Therapeutic Efficiency of Magnetic Hyperthermia in Xenograft Models Achieved with Moderate Temperature Dosages in the Tumor Area. Pharmaceutical Research. 2014;31(12):3274-88. 
14. Kossatz S, Grandke J, Couleaud P, Latorre A, Aires A, Crosbie-Staunton K, et al. Efficient treatment of breast cancer xenografts with multifunctionalized iron oxide nanoparticles combining magnetic hyperthermia and anti-cancer drug delivery. Breast Cancer Research. 2015;17(1):66.

15. Ling Y, Tang X, Wang F, Zhou X, Wang R, Deng L, et al. Highly efficient magnetic hyperthermia ablation of tumors using injectable polymethylmethacrylateFe3O4. RSC Advances. 2017;7(5):2913-8.

16. Bae KH, Park M, Do MJ, Lee N, Ryu JH, Kim GW, et al. Chitosan Oligosaccharide-Stabilized Ferrimagnetic Iron Oxide Nanocubes for Magnetically Modulated Cancer Hyperthermia. ACS Nano. 2012;6(6):5266-73.

17. Arriortua OK, Garaio E, Herrero de la Parte B, Insausti M, Lezama L, Plazaola $\mathrm{F}$, et al. Antitumor magnetic hyperthermia induced by RGD-functionalized $\mathrm{Fe}(3) \mathrm{O}(4)$ nanoparticles, in an experimental model of colorectal liver metastases. Beilstein Journal of Nanotechnology. 2016;7:1532-42.

18. Johannsen M, Thiesen B, Jordan A, Taymoorian K, Gneveckow U, Waldöfner $\mathrm{N}$, et al. Magnetic fluid hyperthermia (MFH)reduces prostate cancer growth in the orthotopic Dunning R3327 rat model. The Prostate. 2005;64(3):283-92.

19. Melanie K, Heike R, Frank W, Sibylle B-S, Lutz T, Werner Alois K, et al. Minimal-invasive magnetic heating of tumors does not alter intra-tumoral nanoparticle accumulation, allowing for repeated therapy sessions: an in vivo study in mice. Nanotechnology. 2011;22(50):505102.

20. Kolosnjaj-Tabi J, Di Corato R, Lartigue L, Marangon I, Guardia P, Silva AKA, et al. Heat-Generating Iron Oxide Nanocubes: Subtle "Destructurators" of the Tumoral Microenvironment. ACS Nano. 2014;8(5):4268-83.

21. Ohtake M, Umemura M, Sato I, Akimoto T, Oda K, Nagasako A, et al. Hyperthermia and chemotherapy using $\mathrm{Fe}(\mathrm{Salen})$ nanoparticles might impact glioblastoma treatment. Scientific Reports. 2017;7:42783.

22. Sandre O, Genevois C, Garaio E, Adumeau L, Mornet S, Couillaud F. In Vivo Imaging of Local Gene Expression Induced by Magnetic Hyperthermia. Genes. 2017;8(2).

23. Lin W, Xie X, Yang Y, Fu X, Liu H, Yang Y, et al. Thermosensitive magnetic liposomes with doxorubicin cell-penetrating peptides conjugate for enhanced and targeted cancer therapy. Drug Delivery. 2016;23(9):3436-43.

24. Yi G-q, Gu B, Chen L-k. The safety and efficacy of magnetic nano-iron hyperthermia therapy on rat brain glioma. Tumor Biology. 2014;35(3):2445-9.

25. Yin PT, Shah S, Pasquale NJ, Garbuzenko OB, Minko T, Lee K-B. Stem cellbased gene therapy activated using magnetic hyperthermia to enhance the treatment of cancer. Biomaterials. 2016;81:46-57.

26. Zhang Z-Q, Song S-C. Thermosensitive/superparamagnetic iron oxide nanoparticle-loaded nanocapsule hydrogels for multiple cancer hyperthermia. Biomaterials. 2016;106:13-23.

27. Zhang Z-Q, Song S-C. Multiple hyperthermia-mediated release of TRAIL/SPION nanocomplex from thermosensitive polymeric hydrogels for combination cancer therapy. Biomaterials. 2017;132:16-27.

28. Brusentsov NA, Nikitin LV, Brusentsova TN, Kuznetsov AA, Bayburtskiy FS, Shumakov LI, et al. Magnetic fluid hyperthermia of the mouse experimental tumor. Journal of Magnetism and Magnetic Materials. 2002;252:378-80.

29. Yang Y, Wang F, Zheng K, Deng L, Yang L, Zhang N, et al. Injectable PLGA/Fe3O4 implants carrying cisplatin for synergistic magnetic hyperthermal ablation of rabbit VX2 tumor. Plos One. 2017;12(5):e0177049. 
30. Liu XL, Yang Y, Ng CT, Zhao LY, Zhang Y, Bay BH, et al. Magnetic Vortex Nanorings: A New Class of Hyperthermia Agent for Highly Efficient In Vivo Regression of Tumors. Advanced Materials. 2015;27(11):1939-44.

31. Cheng Y, Muroski ME, Petit DCMC, Mansell R, Vemulkar T, Morshed RA, et al. Rotating magnetic field induced oscillation of magnetic particles for in vivo mechanical destruction of malignant glioma. Journal of Controlled Release. 2016;223:75-84.

32. Li T-J, Huang C-C, Ruan P-W, Chuang K-Y, Huang K-J, Shieh D-B, et al. In vivo anti-cancer efficacy of magnetite nanocrystal - based system using locoregional hyperthermia combined with 5-fluorouracil chemotherapy. Biomaterials. 2013;34(32):7873-83.

33. Hayashi K, Nakamura M, Miki H, Ozaki S, Abe M, Matsumoto $\mathrm{T}$, et al. Magnetically Responsive Smart Nanoparticles for Cancer Treatment with a Combination of Magnetic Hyperthermia and Remote-Control Drug Release. Theranostics. 2014;4(8):834-44.

34. Haghniaz R, Umrani RD, Paknikar KM. Hyperthermia mediated by dextrancoated $\mathrm{La}(0.7) \mathrm{Sr}(0.3) \mathrm{MnO}(3)$ nanoparticles: in vivo studies. International Journal of Nanomedicine. 2016;11:1779-91.

35. Espinosa A, Di Corato R, Kolosnjaj-Tabi J, Flaud P, Pellegrino T, Wilhelm C. Duality of Iron Oxide Nanoparticles in Cancer Therapy: Amplification of Heating Efficiency by Magnetic Hyperthermia and Photothermal Bimodal Treatment. ACS Nano. 2016;10(2):2436-46.

36. Chen Y, Jiang L, Wang R, Lu M, Zhang Q, Zhou Y, et al. Injectable Smart Phase-Transformation Implants for Highly Efficient In Vivo Magnetic-Hyperthermia Regression of Tumors. Advanced Materials. 2014;26(44):7468-73.

37. Baldi G, Ravagli C, Mazzantini F, Loudos G, Adan J, Masa M, et al. In vivo anticancer evaluation of the hyperthermic efficacy of anti-human epidermal growth factor receptor-targeted PEG-based nanocarrier containing magnetic nanoparticles. International Journal of Nanomedicine. 2014;9:3037-56.

38. Ding Q, Liu D, Guo D, Yang F, Pang X, Che R, et al. Shape-controlled fabrication of magnetite silver hybrid nanoparticles with high performance magnetic hyperthermia. Biomaterials. 2017;124:35-46.

39. Wang F, Yang Y, Ling Y, Liu J, Cai X, Zhou X, et al. Injectable and thermally contractible hydroxypropyl methyl cellulose/Fe3O4 for magnetic hyperthermia ablation of tumors. Biomaterials. 2017;128:84-93.

40. Sadhukha T, Wiedmann TS, Panyam J. Inhalable magnetic nanoparticles for targeted hyperthermia in lung cancer therapy. Biomaterials. 2013;34(21):5163-71.

41. Guo Y, Zhang Y, Ma J, Li Q, Li Y, Zhou X, et al. Light/magnetic hyperthermia triggered drug released from multi-functional thermo-sensitive magnetoliposomes for precise cancer synergetic theranostics. Journal of Controlled Release. 2018;272:145-58.

42. Gao W, Zheng Y, Wang R, Chen H, Cai X, Lu G, et al. A smart, phase transitional and injectable DOX/PLGA-Fe implant for magnetic-hyperthermia-induced synergistic tumor eradication. Acta Biomaterialia. 2016;29:298-306.

43. Verde EL, Landi GT, Gomes JA, Sousa MH, Bakuzis AF. Magnetic hyperthermia investigation of cobalt ferrite nanoparticles: Comparison between experiment, linear response theory, and dynamic hysteresis simulations. Journal of Applied Physics. 2012;111(12):123902.

44. Chatterjee J, Bettge M, Haik Y, Jen Chen C. Synthesis and characterization of polymer encapsulated $\mathrm{Cu}-\mathrm{Ni}$ magnetic nanoparticles for hyperthermia applications. Journal of Magnetism and Magnetic Materials. 2005;293(1):303-9. 
45. Ban I, Stergar J, Drofenik M, Ferk G, Makovec D. Synthesis of copper-nickel nanoparticles prepared by mechanical milling for use in magnetic hyperthermia. Journal of Magnetism and Magnetic Materials. 2011;323(17):2254-8.

46. Laurent S, Dutz S, Häfeli UO, Mahmoudi M. Magnetic fluid hyperthermia: Focus on superparamagnetic iron oxide nanoparticles. Advances in Colloid and Interface Science. 2011;166(1):8-23.

47. Ruiz A, Gutierrez L, Caceres-Velez PR, Santos D, Chaves SB, Fascineli ML, et al. Biotransformation of magnetic nanoparticles as a function of coating in a rat model. Nanoscale. 2015;7(39):16321-9.

48. Gutiérrez L, Lázaro FJ. Comparative study of iron-containing haematinics from the point of view of their magnetic properties. Journal of Magnetism and Magnetic Materials. 2007;316(2):136-9.

49. Martin M, Rodriguez-Nogales A, Garces V, Galvez N, Gutierrez L, Galvez J, et al. Magnetic study on biodistribution and biodegradation of oral magnetic nanostructures in the rat gastrointestinal tract. Nanoscale. 2016;8(32):15041-7.

50. Bobo D, Robinson KJ, Islam J, Thurecht KJ, Corrie SR. Nanoparticle-Based Medicines: A Review of FDA-Approved Materials and Clinical Trials to Date. Pharmaceutical Research. 2016;33(10):2373-87.

51. Lu Y, Xu Y-J, Zhang G-b, Ling D, Wang M-q, Zhou Y, et al. Iron oxide nanoclusters for $\mathrm{T} 1$ magnetic resonance imaging of non-human primates. Nature Biomedical Engineering. 2017;1(8):637.

52. Ito M, Hiramatsu H, Kobayashi K, Suzue K, Kawahata M, Hioki K, et al. $\mathrm{NOD} / \mathrm{SCID} / \gamma$ mouse: an excellent recipient mouse model for engraftment of human cells. Blood. 2002;100(9):3175-82.

53. Roca AG, Morales MP, O'Grady K, Serna CJ. Structural and magnetic properties of uniform magnetite nanoparticles prepared by high temperature decomposition of organic precursors. Nanotechnology. 2006;17(11):2783.

54. Hugounenq P, Levy M, Alloyeau D, Lartigue L, Dubois E, Cabuil V, et al. Iron Oxide Monocrystalline Nanoflowers for Highly Efficient Magnetic Hyperthermia. The Journal of Physical Chemistry C. 2012;116(29):15702-12.

55. Massart R. Preparation of aqueous magnetic liquids in alkaline and acidic media. IEEE Transactions on Magnetics. 1981;17(2):1247-8.

56. Vergés MA, Costo R, Roca AG, Marco JF, Goya GF, Serna CJ, et al. Uniform and water stable magnetite nanoparticles with diameters around the monodomainmultidomain limit. Journal of Physics D: Applied Physics. 2008;41(13):134003.

57. França A, Pelaz B, Moros M, Sánchez - Espinel C, Hernández A, Fernández López C, et al. Sterilization matters: consequences of different sterilization techniques on gold nanoparticles. Small. 2010;6(1):89-95.

58. Talamini L, Violatto MB, Cai Q, Monopoli MP, Kantner K, Krpetić Ze, et al. Influence of size and shape on the anatomical distribution of endotoxin-free gold nanoparticles. ACS nano. 2017;11(6):5519-29.

59. Mehdaoui B, Tan RP, Meffre A, Carrey J, Lachaize S, Chaudret B, et al. Increase of magnetic hyperthermia efficiency due to dipolar interactions in lowanisotropy magnetic nanoparticles: Theoretical and experimental results. Physical Review B. 2013;87(17):174419.

60. Conde-Leboran I, Baldomir D, Martinez-Boubeta C, Chubykalo-Fesenko O, del Puerto Morales M, Salas G, et al. A Single Picture Explains Diversity of Hyperthermia Response of Magnetic Nanoparticles. The Journal of Physical Chemistry C. 2015;119(27):15698-706. 
61. Atkinson WJ, Brezovich IA, Chakraborty DP. Usable frequencies in hyperthermia with thermal seeds. IEEE Transactions on Biomedical Engineering. 1984(1):70-5.

62. Hergt R, Dutz S. Magnetic particle hyperthermia-biophysical limitations of a visionary tumour therapy. Journal of Magnetism and Magnetic Materials. 2007;311(1):187-92.

63. Chu M, Shao Y, Peng J, Dai X, Li H, Wu Q, et al. Near-infrared laser light mediated cancer therapy by photothermal effect of $\mathrm{Fe} 3 \mathrm{O} 4$ magnetic nanoparticles. Biomaterials. 2013;34(16):4078-88.

64. Javed Y, Lartigue L, Hugounenq P, Vuong QL, Gossuin Y, Bazzi R, et al. Biodegradation mechanisms of iron oxide monocrystalline nanoflowers and tunable shield effect of gold coating. Small. 2014;10(16):3325-37.

65. Zitvogel L, Pitt JM, Daillère R, Smyth MJ, Kroemer G. Mouse models in oncoimmunology. Nature Reviews Cancer. 2016;16(12):759-73.

66. Moy AJ, Tunnell JW. Combinatorial immunotherapy and nanoparticle mediated hyperthermia. Advanced Drug Delivery Reviews. 2017;114:175-83.

67. Ito A, Tanaka K, Kondo K, Shinkai M, Honda H, Matsumoto K, et al. Tumor regression by combined immunotherapy and hyperthermia using magnetic nanoparticles in an experimental subcutaneous murine melanoma. Cancer Science. 2003;94(3):30813.

68. Wilhelm S, Tavares AJ, Dai Q, Ohta S, Audet J, Dvorak HF, et al. Analysis of nanoparticle delivery to tumours. Nature Reviews Materials. 2016;1:16014.

69. Jain RK, Stylianopoulos T. Delivering nanomedicine to solid tumors. Nature reviews Clinical oncology. 2010;7(11):653-64.

70. Kherlopian AR, Song T, Duan Q, Neimark MA, Po MJ, Gohagan JK, et al. A review of imaging techniques for systems biology. BMC Systems Biology. 2008;2(1):74.

71. Dähring H, Grandke J, Teichgräber U, Hilger I. Improved hyperthermia treatment of tumors under consideration of magnetic nanoparticle distribution using micro-CT imaging. Molecular Imaging and Biology. 2015;17(6):763-9.

72. Hannecart A, Stanicki D, Vander Elst L, Muller RN, Lecommandoux S, Thévenot $\mathrm{J}$, et al. Nano-thermometers with thermo-sensitive polymer grafted USPIOs behaving as positive contrast agents in low-field MRI. Nanoscale. 2015;7(8):3754-67. 